\title{
Acreditación de unidades docentes multiprofesionales para la formación de especialistas en ciencias de la salud
}

\author{
M. Dolores Vicent-García
}

Introducción. Desde el año 2010, el ministerio competente está acreditando unidades docentes multiprofesionales (UDM) para la formación de residentes médicos, enfermeros y psicólogos en especialidades de campos afines (atención familiar y comunitaria, geriatría, pediatría, obstetricia y ginecología, salud laboral y salud mental) en toda España.

Objetivo. Describir la experiencia y los resultados de acreditación de las UDM, en la Comunidad de Madrid, entre los años 2010 y 2013.

Materiales y métodos. Se ha realizado un estudio descriptivo transversal cuya población de estudio son las UDM de campos afines.

Resultados. Se han acreditado 38 UDM y 516 plazas para la formación de residentes médicos, enfermeros y psicólogos, distribuidas de modo desigual en cinco campos afines.

Conclusiones. Los resultados en términos de acreditación de las UDM son positivos, si bien es necesario continuar explicando entre los profesionales implicados los objetivos que persiguen y evaluar su impacto sobre la formación de especialistas.

Palabras clave. Educación médica posgraduada. Enfermeras residentes. Formación de especialistas. MIR. Residencia. Unidades docentes multiprofesionales.

\section{Accreditation of multi-professional teaching units for specialists training in health sciences}

Introduction. Since 2010 the Ministry of Health is accrediting multi-professional teaching units (UDMs) for training of graduate physicians, nurses and psychologists in field-related specialties (Family and Community Care, Geriatrics, Pediatrics, Obstetrics and Gynecology, Occupational Health and Mental Health) in Spain.

Aim. To describe the experience and results of multi-professional teaching units accreditation in the Community of Madrid, between 2010 and 2013.

Materials and methods. A transversal descriptive study has been made with field-related UDMs as the target population.

Results. 38 multi-professional teaching units and 516 residency places has been accredited for training of graduate physicians, nurses and psychologists unevenly distributed among the five field-related specialties.

Conclusions. The process of accreditation of UDMs has rendered positive results but it seems necessary to clarify the training purposes of UDMs among involved professionals and to assess the impact of UDMs in specialists training.

Key words. Graduate medical education. Multi-professional teaching units. Postgraduate specialists training. Residency. Resident nurse. Resident physician.

\section{Introducción}

Hasta el año 2010, en España, los médicos especialistas y enfermeros especialistas se formaban en diferentes unidades docentes acreditadas -sin actividades conjuntas ni coordinación-, aunque compartiendo escenarios docentes en los mismos servicios asistenciales. A partir de ese año, coincidiendo con la aprobación de los nuevos programas de especialidades de enfermería y el establecimiento de un procedimiento específico de aplicación en todo el país, la Comunidad de Madrid adoptó la decisión de solicitar la acreditación de las unidades docentes multiprofesionales (UDM) para la formación de médicos especialistas y enfermeros especialistas, en el ámbito de la atención familiar y comunitaria, la pediatría y la salud mental, donde también se realiza la formación de psicólogos. El objetivo principal de este trabajo es describir la experiencia de la puesta en marcha de las UDM para la formación de especialistas en la Comunidad de Madrid y los resultados de acreditación obtenidos, entre los años 2010 y 2013.
Consejería de Sanidad. Comunidad de Madrid. Madrid, España.

Correspondencia:

Dña. M. Dolores Vicent García.

Consejería de Sanidad. Comunidad de Madrid. Aduana, 29. E-28013 Madrid.

E-mail:

dolores.vicent@salud.madrid.org

Conflicto de intereses: No declarado.

Competing interests: None declared.

(c) 2015 FEM 
Figura 1. Evolución del número de unidades docentes multiprofesionales por campos afines. AFyC: atención familiar y comunitaria. a La reducción se debe a la fusión de algunas unidades docentes multiprofesionales.

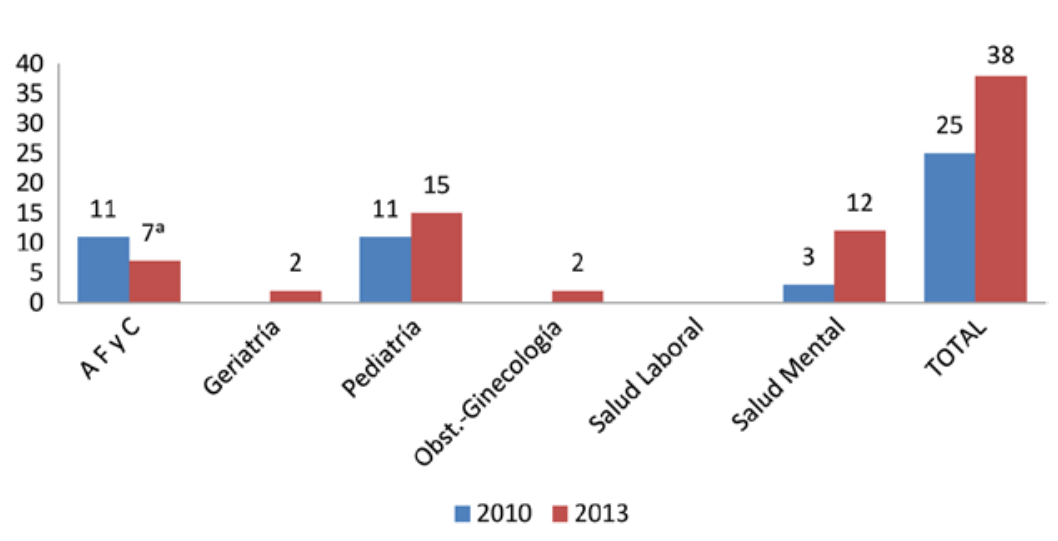

Tabla I. Unidades docentes multiprofesionales cuyas especialidades inciden en campos afines.

\begin{tabular}{lll}
\hline Atención familiar & Especialidad & Titulación de acceso \\
\hline y comunitaria & Medicina Familiar y Comunitaria & Medicina \\
\cline { 2 - 3 } Geriatría & Eeriatría & Enfermería \\
\cline { 2 - 3 } & Enfermería Familiar y Comunitaria & Medicina \\
\hline Pediatría & Pediatría & Enfermería \\
\cline { 2 - 3 } & Enfermería Pediátrica & Medicina \\
\hline Obstetricia y ginecología & Obstetricia y Ginecología & Enfermería \\
\cline { 2 - 3 } Salud laboral & Enfermería Obstétrico-Ginecológica (matrona) & Enfermería \\
\hline Medicina del Trabajo & Medicina \\
\cline { 2 - 3 } & Enfermería del Trabajo & Enfermería \\
\hline Psiquiatría & Medicina \\
\hline Enfermería de Salud Mental & Enfermería \\
\hline Psicología Clínica & Psicología \\
\hline
\end{tabular}

\section{Materiales y métodos}

Se ha realizado un análisis descriptivo transversal, cuya población de estudio son las UDM en las que se desarrolla la formación de especialidades que inciden en campos afines (Tabla I), según lo estableci- do en el anexo II del Real Decreto (RD) 183/2008, de 8 de febrero, por el que se determinan y clasifican las especialidades en ciencias de la salud y se desarrollan determinados aspectos del sistema de formación sanitaria especializada [1]. En su artículo 4 , el RD 183/2008 define las unidades docentes como un 'conjunto de recursos personales y materiales, pertenecientes a los dispositivos asistenciales, docentes, de investigación o de cualquier otro carácter que, con independencia de su titularidad, se consideren necesarios para impartir formación reglada en especialidades en ciencias de la salud por el sistema de residencia, de acuerdo a los establecido en los programas oficiales de las distintas especialidades'. Asimismo, en su artículo 7, regula las UDM estableciendo una distinción entre las unidades docentes en las que distintos graduados se forman en una misma especialidad multidisciplinar y las incluidas en el anexo II, en las que distintos profesionales, (médicos, enfermeras y psicólogos) se forman en especialidades que inciden en campos afines (Tabla I). El estudio se ha efectuado sobre todas las UDM del ámbito de la Comunidad de Madrid, durante el periodo 2010-2013. Las variables del estudio son: la titulación de grado, el tipo de unidad docente, los campos afines y las plazas de formación acreditadas. Los datos sobre los resultados de acreditación de las UDM, las especialidades y las plazas se han obtenido de las convocatorias de pruebas selectivas de 2010 y 2013 para acceso a plazas de formación sanitaria especializada, publicadas en el Boletín Oficial del Estado [2-5].

\section{Acciones para la acreditación de UDM en la Comunidad de Madrid}

Las UDM se regularon en el RD 183/2008 [1] con el objetivo de potenciar el aprendizaje para el trabajo multidisciplinar y multiprofesional, entre otros. No obstante, hasta el año 2010 no se publicaron, por parte del Ministerio de Sanidad, Política Social e Igualdad, los programas oficiales de las especialidades afectadas ni los nuevos requisitos para crearlas y acreditarlas. El escaso tiempo establecido para solicitar la acreditación e incluir las nuevas UDM en la convocatoria anual de plazas de formación sanitaria especializada fue la primera dificultad para iniciar la puesta en marcha de las UDM. La decisión de la Consejería de Sanidad de la Comunidad de Madrid de promover las UDM se ejecutó con el impulso de la Agencia Laín Entralgo, como órgano competente en materia de formación sanitaria especializada, cuyas principales actuaciones fueron: 
- Reuniones informativas y de coordinación institucional con las direcciones generales de atención primaria y hospitalaria, gerentes o directores médicos y de enfermería, y jefes de estudios de hospitales y unidades docentes.

- Asesoría para la elaboración de los expedientes de acreditación, itinerarios formativos, constitución de comisiones y subcomisiones de docencia, etc.

- Revisión de los expedientes de acreditación, realización de los informes preceptivos y tramitación al Ministerio de Sanidad, Política Social e Igualdad.

- Plan de formación multiprofesional de tutores y curso específico para tutores de enfermería.

- Realización de la oferta de plazas para la formación de especialistas de la convocatoria 2010-2011.

\section{Resultados}

Hasta el año 2013, se han acreditado un total de 38 UDM, un 52\% más que en 2010 (Fig. 1). Las UDM acreditadas hasta la fecha constituyen el 62,3\% del total de unidades docentes acreditadas para la formación de las especialidades de campos afines. El avance hacia la acreditación de las UDM ha sido desigual, alcanzando el 100\% de las UDM en atención familiar y comunitaria y sólo el 16,6\% de las UDM en obstetricia-ginecología y el $0 \%$ en salud laboral (Fig. 2).

El número total de plazas acreditadas en UDM asciende a 516, lo que implica un crecimiento global del 26,04\% respecto al año 2010 (Tabla II). Asimismo, las plazas acreditadas en UDM, en el año 2013, suponen el 77,6\% del total de plazas acreditadas de las especialidades de campos afines. Con respecto a la distribución por titulaciones, cabe destacar que las plazas para psicólogos ya alcanzan el 84,6\%; las de médicos, el 77,6\%, y las de enfermería, el 76,5\% (Tabla III).

\section{Discusión}

Ya en el año 2010, los resultados obtenidos en términos de acreditación fueron muy positivos, probablemente por el empuje de los órganos de dirección de la Consejería de Sanidad. Además, en el caso de atención familiar y comunitaria y en pediatría, estos resultados se vieron favorecidos porque las anteriores unidades docentes de medicina familiar y comunitaria se encontraban a su vez en un proceso de reacreditación por los cambios de su programa formativo y se aprovechó la oportunidad
Figura 2. Distribución de unidades docentes (UD) y unidades docentes multiprofesionales (UDM) de especialidades que inciden en campos afines (año 2013). AFyC: atención familiar y comunitaria.

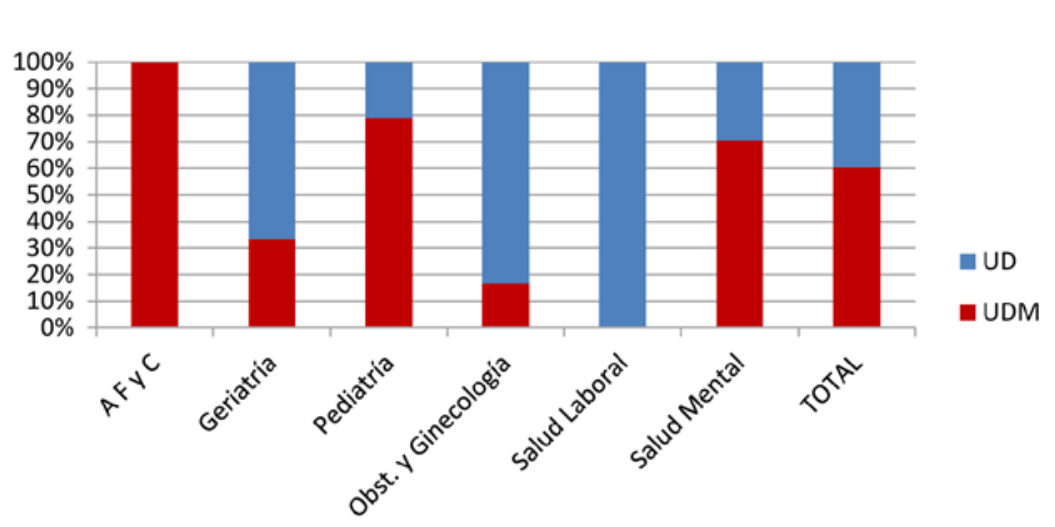

Tabla II. Evolución del número de plazas acreditadas en unidades docentes multiprofesionales según la titulación de acceso.

\begin{tabular}{|c|c|c|c|c|c|c|c|c|}
\hline & \multicolumn{2}{|c|}{ Médicos } & \multicolumn{2}{|c|}{ Enfermeras } & \multicolumn{2}{|c|}{ Psicólogos } & \multicolumn{2}{|c|}{ Total } \\
\hline & 2010 & 2013 & 2010 & 2013 & 2010 & 2013 & 2010 & 2013 \\
\hline Atención familiar y comunitaria & 238 & 238 & 49 & 49 & - & - & 287 & 287 \\
\hline Geriatría & - & 3 & - & 3 & - & - & - & 6 \\
\hline Pediatría & 75 & 81 & 33 & 37 & - & - & 108 & 118 \\
\hline Obstetricia y ginecología & - & 7 & - & 9 & - & - & - & 9 \\
\hline Salud laboral & - & - & - & - & - & - & - & - \\
\hline Salud mental & 10 & 35 & 11 & 32 & 7 & 22 & 28 & 89 \\
\hline Total & 322 & 364 & 93 & 130 & 7 & 22 & 423 & 516 \\
\hline
\end{tabular}

para incorporar la multiprofesionalidad. También se sumó la presión del colectivo de enfermería para poner en marcha sus nuevas especialidades de enfermería familiar y comunitaria y de enfermería pediátrica, lo que necesariamente requería la acreditación de las UDM.

Entre las causas que pueden explicar el bajo porcentaje de reconversión de las unidades docentes de obstetricia-ginecología destaca la existencia previa de unidades docentes acreditadas tanto para médicos como para matronas, y la particular estructura de estas últimas. En la Comunidad de Madrid existe una potente unidad docente de carácter multicéntrico en red para la formación de matro- 
Tabla III. Plazas acreditadas en unidades docentes multiprofesionales (UDM) sobre el total de plazas acreditadas en especialidades de campos afines por titulación.

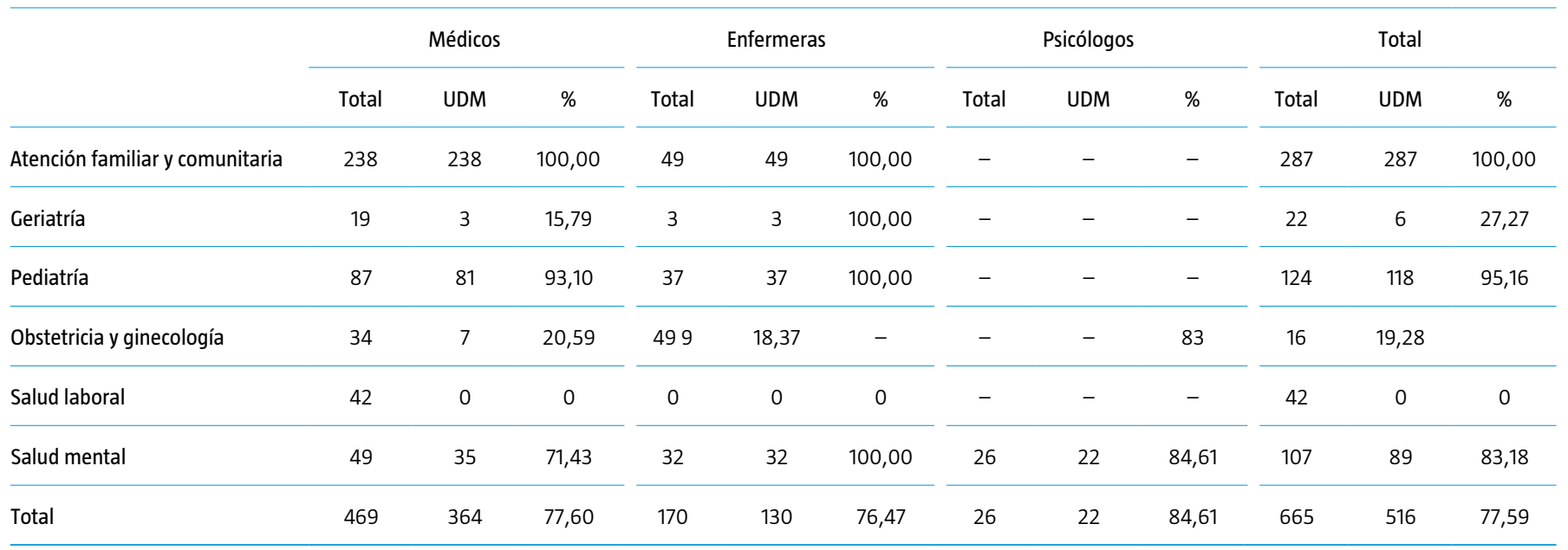

nas, que reúne a la mayoría de hospitales acreditados para esta especialidad, con una estructura de gestión de la docencia independiente y que ha estado vinculada a una escuela universitaria de enfermería, dada la carga teórica de la especialidad. Sin duda, la reconversión de esta unidad docente se vislumbra compleja, ya que exige el cese de su actividad, la pérdida de la comisión de docencia propia y la acreditación simultánea de los hospitales que la componen como UDM hospitalarias, entre otras cuestiones. En cuanto a la salud laboral, las dos unidades docentes de la Comunidad de Madrid son unidades multicéntricas en red, que requerían un cambio estructural para su fusión. En el momento actual se ha solicitado su acreditación como UDM.

A pesar de los positivos resultados en la acreditación, el desarrollo operativo para la puesta en marcha de las UDM no estuvo exento de dificultades porque las normas de acreditación no modifican la cultura de la organización ni los estereotipos existentes sobre los diferentes colectivos. Como era lógico, fueron aflorando ciertas resistencias corporativas ante el cambio iniciado $y$, probablemente por ello, se produjo una significativa falta de liderazgo, sobre todo en mandos intermedios. Además, no se habilitaron nuevos recursos y se pusieron en evidencia las dificultades para establecer el diálogo y la necesaria coordinación entre los miembros de las diferentes profesiones, en pugna por la preservación de su autonomía o por la conservación de su estatus [6]. Así pues, la creación de UDM para la formación de diferentes especialistas fue percibida por algunos profesionales como una imposición y el rechazo se manifestó mediante el cuestionamien- to de la pertinencia de la medida y una resistencia activa a la aplicación de los cambios en el día a día de la formación, que aún persiste en algunas UDM a pesar de la integración estructural realizada en ellas. Asimismo, el apoyo y la tutela institucional que deberieron haberse desarrollado desde la Agencia Laín Entralgo se vieron interrumpidos por el cierre de ésta en el año 2012, en el marco de la adopción de medidas urgentes para la racionalización del gasto público.

A pesar de todo, en los tres años de desarrollo de las UDM, los resultados de acreditación han continuado mejorando por la iniciativa de los responsables de determinados servicios asistenciales, que han sabido ver en las UDM una oportunidad de mejora en la formación de los especialistas. Además, se observan otros aspectos positivos porque en numerosas UDM se están realizando actividades de formación conjuntas, para todos los residentes, y con ello, más actividades en las que profesionales de distinta titulación, ya especialistas, trabajan en equipo, colaborando para conseguir un objetivo común.

Del análisis de la experiencia descrita se puede concluir que los resultados de la acreditación de UDM entre 2010 y 2013 han sido muy favorables, si bien se necesita más información sobre el propósito de su implantación entre los profesionales implicados. Asimismo, son necesarias distintas intervenciones formativas orientadas a la adquisición de los conocimientos, habilidades y actitudes, necesarios para el desarrollo del ejercicio multidisciplinar y multiprofesional, no sólo en la formación de especialistas, sino en todos los niveles formativos [7]. Por último, se estima conveniente la evaluación del im- 
pacto de la implantación de las UDM sobre la formación de las profesiones de especialidades que inciden en campos afines y la elaboración de un plan de mejora en función de los resultados obtenidos.

\section{Bibliografía}

1. Real Decreto $183 / 2008$, de 8 de febrero, por el que se determinan y clasifican las especialidades en ciencias de la salud y se desarrollan determinados aspectos del sistema de formación sanitaria especializada. Boletín Oficial del Estado, n. ${ }^{\circ} 45$, de 21 de febrero de 2008.

2. Orden SAS $/ 2448 / 2010$, de 15 de septiembre, por la que se aprueba la convocatoria de pruebas selectivas 2010 para el acceso, en el año 2011, a plazas de formación sanitaria especializada para médicos, farmacéuticos y otros graduados/ licenciados universitarios del ámbito de la psicología, la química, la biología y la física. Boletín Oficial del Estado, n. ${ }^{\circ} 230$, de 22 de septiembre de 2013.

3. Orden SSI/1695/2013, de 12 de septiembre, por la que se aprueba la convocatoria de pruebas selectivas 2013 para el acceso, en el año 2014, a plazas de formación sanitaria especializada para médicos, farmacéuticos y otros graduados/ licenciados universitarios del ámbito de la psicología, la química, la biología y la física. Boletín Oficial del Estado, n. ${ }^{\circ} 228$, de 23 de septiembre de 2013.

4. Orden SAS/2447/2010, de 15 de septiembre, por la que se aprueba la convocatoria de prueba selectiva 2010, para el acceso en el año 2011, a plazas de formación sanitaria especializada para graduados/diplomados en enfermería. Boletín Oficial del Estado, n. ${ }^{\circ}$ 230, de 22 de septiembre de 2013.

5. Orden SSI/1694/2013, de 12 de septiembre, por la que se aprueba la convocatoria de prueba selectiva 2013, para el acceso en el año 2014, a plazas de formación sanitaria especializada para graduados/diplomados en enfermería. Boletín Oficial del Estado, n. ${ }^{\circ} 228$, de 23 de septiembre de 2013.

6. Vicent MD. Unidades docentes multiprofesionales en la Comunidad de Madrid. Jornada 'Las nuevas unidades multiprofesionales y avances en metodología docente'. Organización Médica Colegial, 4 de abril de 2011. URL: http://www.ffis.es/areda/evento02.

7. Coll J, Bernabeu D, Cervantes C, Nolla M, Muniesa JM, Tor J, et al. Un modelo de aprendizaje multiprofesional en ciencias de la salud: innovación docente como respuesta a las necesidades emergentes de nuestra sociedad. Educ Med 2010; 13 (Supl 1): S13-9. 